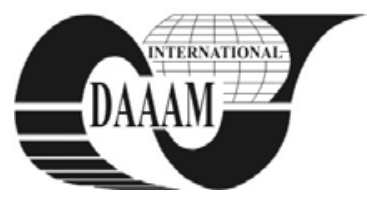

\title{
A METHODOLOGICAL FRAMEWORK FOR KNOWLEDGE CAPITALIZATION IN ACCOUNTING AND FINANCIAL AREA
}

\author{
VRINCIANU, M[arinela]
}

\begin{abstract}
In accounting information systems, most research on knowledge management assumes that knowledge has positive implications for gaining competitive advantage. This research attempts to identify the specific issues that must be taken into account when designing an organizational memory information system (OMIS) for financial and accounting domain. This paper suggests a possible methodological framework for capitalization of knowledge and creation of a learning organizational memory in this particular domain.
\end{abstract}

Key words: OMIS, knowledge capitalization, capitalization method

\section{INTRODUCTION}

A possible solution for implementing a knowledge management $(\mathrm{KM})$ project in an accounting organization could be the creation of an OMIS. ICT provides the infrastructure and tools needed to materialize the organizational memory in a variety of forms: a base case, a database, a knowledge base, a documentary base, an interactive system to assist decision, a collaborative tool or a Semantic Web. Identification and capitalization of knowledge is achieved through specific methods of knowledge management.

The interest of organizations in the capitalization of knowledge and in the use of organizational memory systems is justified by: the loss of know-how due to the personnel mobility (retirement, transfer, finding a new job, etc.), sharing tasks and responsibilities on certain jobs, the increasing specialization of accountancy professions and the need for collaborative work.

The emergence of ICT and, in particular, of the Semantic Web technology, generated new approaches in the modelling of accounting information systems. Including the semantic level and attaching the so-called organizational memory system functionalities for training in a conventional accounting information system can directly contribute to the improvement of working procedures. This study attempted to address the following major research questions: (1) what are the perceived needs for training in Romanian accounting organizations? (2) What are the major issues that occur when modelling a learning organizational memory for accounting and financial area? (3) What are the potential stages of development process of an OMIS for this particular domain?

\section{METHODOLOGY}

The first stage of our research focused on the scientific investigation of the main methods of knowledge management used in the construction of organizational memory, structured in three main categories: (1) methods aiming at knowledge capitalization approach, (2) methods concerning the continuous storage of knowledge in order to return the obtained experience and (3) methods of mapping knowledge. Work premises and research objectives were established and validated after reception and analysis of data provided by empirical researches realized in Romanian organizations (Vrîncianu et al., 2009). The total number of distributed questionnaires was 450 and 131 were with usable answers. In the second stage, our research has focused on identifying the categories of knowledge involved in accounting practices, in working procedures and rules. A survey questionnaire based on the application of a set of 25 questions for each accountancy profession (open questions and questions with simple selection) was conducted between 15 October, 2010 and 30 November, 2010. Administration of the questionnaires was done both by direct interview and by e-mail. The most effective results were obtained from direct interviews with financial auditors, which allowed the collection of valuable knowledge based on their personal experiences. In many cases, the interview and the analysis of verbal protocols proved more efficient for identifying key knowledge.

\section{LITERATURE REVIEW}

The use of knowledge modelling methods in particular and of semantic modelling in general has had very important effects in accounting over the last 30 years. The conceptual modelling of various business processes (e.g. REA models) provided taxonomy of conceptual objects and elements to use in describing and teaching the accounting information architecture of typical business enterprises. Literature reveals the inability of classic accounting systems to facilitate non-financial decisions (Dunn \&McCarthy, 1997). Many researches are focused on semantic modelling in accounting: audit planning, models of internal control and models of planning. Modelling learning organizational memory is the subject matter of numerous academic publications and research projects: MEMORAe, Ariadne or Arpem.

Literature reveals various attempts to produce domain ontology for accounting by using semantic modelling: Teller (2008) provided a formal representation of accounting standards (IFRS), Chou et al. (2008) proposed a knowledge management approach in accounting via ontology development and Anica-Popa (2010) analysed the possibility of capitalization of knowledge in financial and accounting area.

\section{RESULTS}

Based on the survey responses, one major finding was that the amount of time devoted to training did not appear to be consistent with how important the needs were. There seems to be a recognized demand for expanded training hours (47\% of respondents), more quality training resources (32\% of respondents) and specialized ICT tools for quick information retrieval (83\% of respondents). Generally speaking, these survey results support the general conclusion that training and knowledge sharing are considered important by accounting organizations, but they are continuing to ignore their internal 
training resources and cognitive potential, seeking outside training firms and generally providing just an initial training to recruits. Without being familiar with the concept of OMIS, about $76 \%$ of respondents accepted the idea that having a system that allows collaboration and sharing of useful knowledge in performing everyday tasks could contribute positively to the creation of firm's value.

The major issues faced when modelling a learning organizational memory for financial and accounting domain are: (1) difficulty of selecting knowledge that will be introduced in a domain ontology because of the complexity of modelling such a diverse content; (2) frequent changes of legal norms, accounting systems and financial standards; (3) the change in the attitude of groups of accountants: the preference for flexibility and less uniformity in financial regulations application; (4) modelling the tacit knowledge; (5) study of heredity (survival of concepts and practices to the change in the accounting system) and thanatogenesis (the causes of the disappearance of accounting concepts or practices) in order to understand why certain accounting concepts disappear, while others endure; (6) existence of an idiomatic accounting language and problems of synonymy/polysemy in accounting or inter-lingual translation; (7) vocabulary changes in Romanian accounting (comparison of accounting data before 1989 with recent data); (8) the existence of formal models that unify accounting and auditing; (9) the heterogeneous character of memory sources.

The proposed methodological framework for knowledge capitalization in financial and accounting area is based on a process that includes the following potential stages: (1) defining competency models of accountancy professions (financial auditor, accountant, accountant expert); (2) gathering the accounting information from different data sources: accounting information systems, working procedures, rules, norms, analysis models, etc. It is possible to use different techniques for gathering knowledge: interviews, analysis of verbal protocols obtained by simulation of problem solving, direct observation, the use of a questionnaire, retrospective verbalizations, introspection, "Wizard of Oz" technique, the use of a collective protocol for gathering knowledge, etc.; (3) analyzing accounting items by classifying them in accordance with three definitions: meaning of item, the relationships between items, the operation of items; (4) organizing vocabulary and constructing of an operational taxonomy (a hierarchical structure to classify information); (5) building the thesaurus that lays out a semantic road map, relating concepts to terms and providing definitions; (6) obtaining conceptual schema that involves specification of the meaning of the explanatory categories for accounting domain; (7) modelling the concepts, the roles, the individuals and their relationships (Description Logic). The fundamental modelling concept of a Description Logic is the axiom - a logical statement relating roles and concepts; (8) obtaining the domain ontology that is a set of defined concepts describing a specific domain, using a subclass hierarchy, by defining properties and relationships; (9) use of ontology is the stage in which one specifies the interaction scenario between the future users and organizational memory, means of dissemination, commonly used informational technologies (such as Internet, Intranet or other), the dissemination approach; (10) the system evaluation supposes the existence of a set of criteria for evaluation, the usage of surveys to gather the views of employees of the organization (usability tests), the use of experts to evaluate results, questionnaires with questions based on Likert scale or checklists.

\section{CONCLUSIONS AND FUTURE DIRECTIONS}

Formalization of knowledge provided by the financial and accounting area and inclusion in single domain ontology is far from being an achievable goal. Even if semantic modelling in accounting has been a research subject for over four decades, the creation of a viable, lasting and efficient system over the long term is still impossible, partly because of a multitude of implied sub domains (financial analysis, cost calculation, primary evidence, taxation, accounting expertise, auditing, etc.) and, on the other hand, different expertise levels of potential users, and distinct specializations. An essential element in designing such a system remains its teleological perspective: training, sharing knowledge, recovery or retrieval, document content management. We consider that challenges to developing successful organizational memory systems follow three major directions: (1) issues related to managing the knowledge, (2) issues related to the people who generate and use the knowledge and (3) challenges related to practices, procedures and methods used to build these systems.

Future work on semantically-modelled accounting systems should proceed on two major directions: (1) the design of new constructs, methods and technics; (2) the creation of new or alternative models.

\section{ACKNOWLEDGEMENTS}

The research reported in this paper was financed by CNCSIS-UEFISCSU as a component of the research project called "Research in the field of modelling and building organizational memory. OMCAAF - a new methodological framework for financial and accounting cognitive Acquis Capitalization”, in the framework of National Plan of Research, Development and Innovation - PN II, Ideas Program, 2008 Competition. (Project number PNII-IDEI Id_1866/2008, Contract number: 766/2009).

\section{REFERENCES}

Anica - Popa, L. - E. (2010). Capitalizing the Cognitive Acquis in the Accounting and Financial Area: A Morphology of the Organizational Memory, Annals of DAAAM for 2010 \& Proceedings of the 21st International DAAAM Symposium, 20-23rd October 2010, Zadar, Croatia, ISSN 1726-9679, ISBN 978-3-901509-73-5, Katalinic, B. (Ed.), pp. 14671468, Published by DAAAM International Vienna, Vienna

Chou T.-H., Vassar J., B. Lin,(2008). Knowledge management via ontology development in accounting. Kybernetes. Vol. 37 Iss: 1 . pp. 36 - 48, ISSN 0368-492X

Dunn C., McCarthy W. (1997). The REA accounting model: intellectual heritage and prospects for progress. Available from:https://www.msu.edu/user/mccarth4/DUNN\&MC.htm Accessed: 2011-08-12

Teller, P.(2008).The use of a formal representation of accounting standards. International Journal of Computer Science and Applications, Vol. 5, No. 3b, pp. 93-116, ISSN 0972-9038

Vrîncianu,M.,Anica-Popa ～L.,Anica-Popa ～I. (2009). Organizational memory: an approach from Knowledge Management and Quality Management of organizational learning perspectives. Amfiteatrul Economic, no.26, June 2009, pp.473-48, ISSN 1582-9146 